\title{
Experiences of psychosocial and programme-related barriers to recovery in lifestyle interventions for noncommunicable diseases
}

\author{
P Skowno, $\mathrm{PhD}^{1}$, w Derman, $\mathrm{MBChB}, \mathrm{FFIMS}, \mathrm{PhD}^{2}$, D J Stein, \\ $\mathrm{MBChB}, \mathrm{FRCPC}, \mathrm{PhD}^{3}$, C E Draper, $\mathrm{PhD}^{1}$ \\ ${ }^{1}$ Division of Exercise Science and Sports Medicine, Department of Human \\ Biology, University of Cape Town, South Africa and Sport Science Institute \\ of South Africa, Newlands, South Africa
}

${ }^{2}$ Department of Orthopaedics, University of Stellenbosch, South Africa

${ }^{3}$ Department of Psychiatry and MRC Unit on Anxiety and Stress Disorders, University of Cape Town, South Africa

\section{Corresponding author: P Skowno (pskowno@gmail.com)}

Background: The majority of global deaths are due to noncommunicable diseases, largely preventable and treatable utilising behavioural interventions.

Objectives: The study investigated patients' experiences of a lifestyle intervention programme for noncommunicable diseases (NCDs), and the influence that psychosocial and programme-related barriers had on patients' ability to improve their well-being.

Methods: Fourteen patients with NCDs were interviewed before and on completion of a 12-week lifestyle intervention programme at a sports and exercise medicine clinic. Thematic analysis techniques were used to analyse interview data.

Results: Patients described their experiences of NCDs diagnosis as traumatic, and their own relationship with their bodies and with the disorder(s) to be vulnerable and significantly challenging. Professional incompetence and unethical treatment were included as barriers to recovery. Barriers specifically relating to the programme included scheduling, as well as the online assessment component. Those reporting more premorbid psychosocial barriers were more likely to experience current complications, whether disease- or treatment-related, often emphasising the negative influence of programme and professional-related problems.

Conclusion: Qualitative methodologies enabled the study to yield clinically relevant insights with respect to patients with NCDs. Accounting for the trauma and vulnerability experienced by this cohort may assist in the development of more patient-centred interventions and sustainable secondary prevention of NCDs.

Keywords: chronic diseases of lifestyle; intervention evaluation; psychological risk factors; social support; professional conduct

S Afr J Sports Med 2016;28(3):69-73. DOI: 10.17159/2078-516X/2016/v28i3a1237

The majority of recent global deaths are due to noncommunicable diseases (NCDs) ${ }^{[1]}$. Many of these diseases are preventable by modifying key risk factors such as unhealthy diet, physical inactivity, tobacco use and harmful alcohol use [1]. However, the complex nature of the disease experience make it unavoidable to dismiss the challenges inherent in sustainable behaviour modification ${ }^{[2]}$. This is demonstrated by the continuing increase in global incidence of NCDs. In turn, the development of more individualised, patient-centred treatment for these cases may require a more comprehensive research strategy to better understand patient experiences of NCDs and their participation in lifestyle intervention programmes. This is often achieved through the application of qualitative research strategies.

The application of qualitative and mixed methodologies in health science research has resulted in a corresponding increase in literature detailing patient experiences of NCDs and the recovery process $^{[3-7]}$ To date, a number of reviews ${ }^{[3]}$ and systematic reviews ${ }^{[4,5]}$ have collated patients' experiences of chronic heart failure, and qualitative research has distinguished generic- and disease-specific experiences of NCDs ${ }^{[6]}$. Elderly patients experience chronic heart failure as debilitating and distressing. There is a great deal of uncertainty, especially at an advanced stage of illness and inefficacy in self-care ${ }^{[4]}$. Social isolation, living in fear, and losing a sense of control were included as prominent themes in a subsequent review of the topic ${ }^{[5]}$. Adaptation to a new sense of self is suggested as influencing self-care behaviours ${ }^{[3]}$. Moreover, patients recovering from myocardial infarctions noted difficulties in making lifestyle changes ${ }^{[7]}$. Accordingly, findings called for increased long-term support and monitoring, as well as group work to enhance the sharing of experiences ${ }^{[7]}$.

A systematic review of patient experiences of chronic heart failure noted the nature of health service encounters as an important factor influencing patient health trajectories ${ }^{[5]}$. It incorporates access, continuity and quality of care, as well as comorbid conditions and personal relationships, and thus plays an important role in developing and improving the delivery and use of lifestyle interventions for patients with NCDs ${ }^{[5]}$.

Research of patient experiences of cardiac rehabilitation highlights the barriers to adherence or attendance. Using qualitative methodologies, a systematic review and metasynthesis of cardiac rehabilitation studies identified physical and personal barriers to participation ${ }^{[8]}$. Examples of physical barriers included a lack of transport and financial constraints. Personal barriers may include a patient feeling embarrassment about participation, or misunderstanding the reasons for the onset of the disease, or the purpose of rehabilitation.

What is less evident, however, are the characteristics and experiences of NCDs' cohorts with multiple comorbidities who are participating in lifestyle intervention programmes. Extensive descriptions of these cohorts could potentially guide the development of more patient-centred interventions.

Therefore the aim of this paper is to describe the role of factors which may hinder a patient's physical and psychological progress in a lifestyle intervention programme for NCDs. Specifically, the paper's objectives are to outline psychosocial as well as programme-related factors which might contribute towards less successful physical and mental health outcomes in patients with NCDs. 


\section{Methods}

\section{Study design}

The study used qualitative methodology. Data collection and analysis involved semi-structured interviews and thematic analysis respectively.

\section{Study setting}

$U$ Turn Medical is a multi-disciplinary, comprehensive lifestyle intervention programme centred on the patient. The 12-week programme is designed to provide optimal health care for patients with a range of established chronic diseases, including cardiovascular disease, metabolic and chronic respiratory disorders. The programme manages established disease states and recognised risk factors, and aims to improve a patient's functional capacity. It provides risk screening, medical assessment, supervised exercise sessions, injury prevention strategies, dietary education and psychosocial support.

Individualised exercise and lifestyle prescriptions are developed for patients during preliminary one-on-one exercise sessions with a biokineticist. Exercise, dietary, and psychosocial prescriptions are based on findings from baseline medical, exercise and psychosocial assessments. The majority of patients subsequently participate in group exercise sessions held three times a week in a morning or afternoon session. The group exercise sessions are supervised by a biokineticist. A sports physician is in attendance to provide a prompt and appropriate response to any potential emergencies during exercise sessions. Prior to completion, patients are reassessed by a biokineticist and a sports physician. This may be followed by an additional 12-week programme if indicated.

\section{Participants}

Participants were identified and recruited from a cohort of patients with NCDs starting the U Turn Medical ${ }^{[9]}$ lifestyle intervention programme. Convenience sampling was deemed the most suitable strategy for the investigation, based on the availability of new intakes into the programme and referral from biokineticists and doctors at clinics. Cases meeting referral criteria for the programme were included in the study. The majority of participants were referred to by programme staff as 'cardiac patients'.

Fourteen individuals consented to being interviewed at the start of the programme. Eleven of the 14 were available for recorded interviews at the end of programme. Two participants submitted written responses due to the reported inconvenience of returning to the study site, and one participant who did not complete the programme did not respond to requests for a follow-up interview. Of the 11 participants interviewed, two withdrew from the programme due to health complications, with one reporting the intention of resuming the programme at the time of the second interview.

An overview of participant characteristics is detailed in Table 1, and reflect similar demographic trends to related patient populations, as reported in related studies ${ }^{[9]}$.

\section{Data collection}

Interviews before and after the intervention were semistructured, consisting of open-ended questions within a flexible structure to define a specific area of inquiry, as well as divergence to another area if necessary ${ }^{[10,11]}$. The questions for both interviews are detailed and italicised in Table 2. Interviews took place in a consulting room at the clinic and recorded using a digital voice recorder. They were conducted by the first author, a registered counselling psychologist, and lasted between 15 and 50 minutes respectively. On average, the initial interview was conducted during the participant's second or third week after the physician's assessment. The second interviews were held any time from the last few weeks of the participant's programme to six months thereafter. The recordings were subsequently transcribed and analysed.

\section{Ethical considerations}

Ethical approval for this study was obtained from the Human Research Ethics Committee of the Faculty of Health Sciences at the University of Cape Town (HREC REF 332/2007) in accordance with the Helsinki Declaration. Participants gave their signed, informed consent.

\section{Data analysis}

Interview data were analysed using thematic analysis, a userfriendly and intuitive method of identifying and selecting central themes and patterns in data ${ }^{[12]}$. It is a flexible method and can be adapted into numerous research designs, irrespective of the researcher's theoretical background ${ }^{[13]}$. Thematic analysis identifies basic features of the data that are of interest to the researcher ${ }^{[12]}$. This is achieved through coding, a process of extracting the most basic, yet meaningful segments of raw data from a data set ${ }^{[12]}$. Following the coding process, themes were identified. Themes relate the important aspects of data to the study's research question ${ }^{[12]}$. They occur relatively frequently within the data set and carry some degree of meaning ${ }^{[12]}$. The study made use of QSR NVivo 10 data analysis software.

After the start of the preliminary analysis, a thematic framework was constructed in which to consolidate similar themes and perceive the differences from others. The themes identified in the data set were categorised as: psychosocial barriers and professional and programme-related barriers to progress in the programme.

\section{Results}

The following section initially describes premorbid and disease-related psychosocial barriers commonly reported by the participants. It subsequently outlines other programmerelated factors participants reported as influential in impeding potential improvements in health. Specifically, the importance of professional and ethical conduct by health practitioners (in the programme and in general) is highlighted, as well as a number of technical issues which may have been perceived as frustrating enough to interfere with progress.

\section{Theme 1: Psychosocial barriers}

Participants were asked whether they believed that any 
Table 1. Demographic and medical characteristics of participants $(\mathrm{n}=14)$

\begin{tabular}{|c|c|c|}
\hline \multirow[t]{2}{*}{ Age (2014) } & $15-59$ & 5 \\
\hline & $60-84$ & 9 \\
\hline Sex & Male: Female & $10: 4$ \\
\hline \multirow[t]{2}{*}{ Race } & White & 12 \\
\hline & Coloured & 2 \\
\hline \multirow[t]{3}{*}{ Employment } & Full time & 8 (all self but 1 ) \\
\hline & Part time & 1 \\
\hline & Retired & 4 \\
\hline \multirow[t]{2}{*}{ Referral to $U$ Turn } & $\begin{array}{l}\text { Fedhealth Medical } \\
\text { Scheme }\end{array}$ & 6 \\
\hline & Cardiologist/ other & 7 \\
\hline Premorbid best & $0-3$ years & 9 \\
\hline $\begin{array}{l}\text { (before diagnosis/ } \\
\text { event) }\end{array}$ & Over 3 years & 5 \\
\hline Interviews & Interview 1 & 14 (in person) \\
\hline completed & Interview 2 & $\begin{array}{l}11 \text { (in person) } \\
2 \text { (written) }\end{array}$ \\
\hline \multirow[t]{3}{*}{$\begin{array}{l}\text { Activities since } U \\
\text { Turn }\end{array}$} & $\begin{array}{l}\text { Completed } \\
\text { programme, then own } \\
\text { gym }\end{array}$ & 4 \\
\hline & $\begin{array}{l}\text { Completed more } \\
\text { programmes }\end{array}$ & 7 \\
\hline & $\begin{array}{l}\text { Did not complete, but } \\
\text { did some exercise }\end{array}$ & 2 \\
\hline \multirow[t]{3}{*}{ Social support } & Married & 9 \\
\hline & Single & 3 \\
\hline & Divorced & 2 \\
\hline \multirow[t]{2}{*}{ Primary diagnosis } & $\begin{array}{l}\text { Cardiovascular } \\
\text { disease }\end{array}$ & 13 \\
\hline & Metabolic disorders & 1 \\
\hline
\end{tabular}

Table 2: Semi-structured interview framework

Interview 1:

- When was the last time you remember feeling your (psychological, physical, spiritual) best?

- What happened since then?

- Detail the events that brought you to the U Turn Programme

- Where you referred by Fedhealth? (if not mentioned)

- What are your expectations of the programme? What do you hope will be achieved?

- (If already commenced) What are your impressions so far of the programme?

- What personal qualities may assist you in successfully completing this programme?

- How would you define the term resilience?

- How does it apply to your life?

- Is there any potential benefit to what has happened to you?

- What aspects of your recovery are within your control? (or not) personal history, relationships or qualities may have hindered their ability to improve their health during the programme. A number of participants reported premorbid and or current psychosocial difficulties. A common theme identified among the comments from the patients included negative responses to the initial diagnoses of NCDs.

\section{Premorbid and current psychosocial barriers}

Psychosocial barriers ranged from childhood traumas to chronic mood disorders and multiple current stressors, all of which complicated the treatment process of those afflicted. Not all accounts of premorbid or current psychosocial problems, however, were indicative of poor progress in the programme. Of the six participants identified as currently experiencing or having a history of psychosocial difficulties, three reported achieving physical progress by the end of the programme. The remaining three, however, experienced continued difficulties in initiating and or maintaining health changes. Current psychosocial stressors involved work, difficult relationships, comorbid conditions and drug side effects which complicated the treatment process, family commitments, and problems related to retirement.

\section{Responses to NCDs}

There were varied reactions by participants to their respective health challenges. For some, the decline in physical condition came as a shock, highlighting a disconnection between perceived and actual health status:

- Correct. I thought, you know, "I'm a fit, healthy guy, and I exercise a lot, and I'm looking after my health, I don't smoke. And here I'm getting a heart transplant. What's that about?" [laughs] (Male, 50 years).

- ...the only adverse thing was that I couldn't bring myself to actually say, "you had a heart attack". And I still don't even like saying it now. (Male, 72 years)

Many participants responded pessimistically to their initial NCD event and diagnosis. For a few, this included blaming themselves for their poor health:

- Ja! And, and this has made me think, "you know what, you are 54, you've buggered around for thirty something years, or forty years, since I've been overweight, taking chances." I mean, some of the weight is, medically... caused, and the other is completely self-induced. I eat too much, I drink too much, I smoke 40 cigarettes a day. Um.. and used to eat every second day to try and control it. So ... I've done all that damage [3 sec pause] Now you've got to a point where you're going to have to [ $3 \mathrm{sec}$ pause] live with what you've got, and get it to be the best... functioning body. Which I've never done to my poor body. Because I've always had so many things wrong with it, it's like, "oh for god's sake something else". (Female, 55 years).

Others experienced a significant loss in confidence, particularly in their bodies. These reactions often resulted in a sense of helplessness and decreased motivation to make the necessary health changes: 
- So, it's my first... feeling of being in a health problem, of having a health problem, and thinking, "I'm now scared if I have a heart attack or something!" You know? Can I push myself? I don't know. (Female, 52 years).

- I am so lacking in confidence in myself I find it even difficult - I went to the shop with my wife earlier today, uh, because I'm not even driving anymore, I'm that uncomfortable you know... I don't recognise this body at all right now... This is definitely the most traumatic event I've had to deal with. (Male, 61 years).

\section{Theme 2: Professional and programme-related problems Professional incompetence}

Several participants reported experiencing dissatisfaction with the healthcare provided by at least one professional, either currently, or in the past. Reasons included unethical conduct or the failure to diagnose and effectively treat certain complications. That said, the professionals concerned were not directly involved with the clinic, or the U Turn Medical programme. All of the above factors had the potential to impede the participants' progression towards healthier outcomes, and even adhering to the programme itself, as experienced by a male participant of 61 years. He dropped out of the programme because of poorly managed, severe side effects from his anticoagulant medication, leading to an overall sense of helplessness and demotivation:

- The feeling is, you know, how does anybody actually know what is going on with this heart of mine? Is it improving or isn't it? You know? (Male, 61 years).

A female participant of 55 years relayed an unpleasant interaction with her cardiologist:

- Um, had an appalling... doctor who said I didn't deserve the heart I had because I was so fat. Um, because I apparently had the heart of a 35 year old and I'm 54 . Um, because there is no narrowing, there's no cholesterol issues, he says, nothing. Um, but... he cleared me for that, discharged me, and now I've just been panicking ever since then. Never saw him again, refused to consult with him. (Female, 55 years).

Another participant communicated her unease with the ability of previous biokineticists in managing certain patient populations. Moreover, a number of participants had complicated presenting problems at programme intake, and communicated a lack of faith in their doctor's ability to correctly manage their conditions.

\section{Administrative and logistical problems}

A few participants found the timing of the programme to be too intensive. They reasoned that three times a week, at over 90 minutes a session, took significant time away from other commitments. The times that were offered were additionally considered to be inadequate and problematic from a commuter's perspective.

Several participants expressed frustration with U Screen, an online assessment and educational component of the programme. Problems included the software, where a number of participants were required to recomplete and resubmit assessments on several occasions. Many participants, although being at least partially computer literate, struggled with accessing and completing the forms from tablets and mobile phones and requested paper versions. Others simply found the educational modules to be unnecessary and time-consuming, as they felt that had sufficient knowledge of the topics covered and did not need to complete the modules.

\section{Discussion}

The study investigated patients' experiences of a lifestyle intervention programme and the influence of psychosocial and programme-related factors on their ability to improve their physical and psychosocial well-being. The most important finding was that nearly all the participants experienced at least a moderate degree of trauma at the time of their initial diagnosis. This occurred in individuals with varying NCDs, as well as premorbid physical and psychosocial well-being. Similar accounts can be found in related research ${ }^{[3-7,14]}$. Furthermore, the impact of earlier psychosocial difficulties, rather than the events themselves, was considered. While not all participants experienced a major cardiac event, most reported that their lives and confidence in their bodies had been significantly altered by the diagnosis. For some, this included a sense of disconnection between perceived and actual health, and for others it resulted in self-blame. While reviews by $\mathrm{Yu}$ et al. and Jeon et al. respectively highlighted the distress and uncertainty experienced by patients living with $\mathrm{CHF}$, both reviews focused on a significantly older population ${ }^{[4,5]}$. Another study of patients recovering from heart attacks identified the uncertainty patients felt between being 'well' and still being 'ill' after hospital discharge, as well as the need for longer-term support in managing prescribed changes in lifestyle ${ }^{[7]}$.

Some participants who reported past psychosocial difficulties complained of having current problems and comorbid conditions. Not all accounts of premorbid difficulties, however, were indicative of poor physical progress during the programme. This serves as another reminder of the multifactorial and subjective nature of psychosocial barriers, and the need for more individualised assessment and management of NCDs ${ }^{[2,15]}$.

Themes relating to professional incompetence were included as a concern for several participants. Specifically, participants experiencing chronic, undiagnosed (or misdiagnosed) symptoms had less faith in the ability of healthcare practitioners to treat their primary NCDs. Moreover, a number of responses relating to professional misconduct emphasised the potentially negative impact practitioners have on their patient's progress. Related findings are reported in NCDs literature, with the nature of health service encounters being reported as an important factor impacting on the ability of patients' with chronic heart failure to manage their disease ${ }^{[5]}$.

Lastly, technical and administrative problems included the 
timing of classes, as well as the electronic and online assessments which were faulty or inaccessible to patients who were not computer literate. A few participants found such problems to be detrimental to their progress. For most patients, the quality of interactions with practitioners and with the programme staff was considered more important to their progress than the above factors. These findings are found in other rehabilitation and NCDs literature, which emphasise the importance of social support and health education in a patient's lives ${ }^{[14]}$.

\section{Limitations}

It is of paramount importance that researchers account for their role as a research instrument in qualitative methodologies. Researchers' personal values and biases may impact the trustworthiness of the collection, analysis and reporting of participant responses.

Moreover, the power dynamics between researcher and participant should always be considered. A number of the participants may have felt pressured into volunteering for the interviews, or disclosing more than usual because the researcher was often presumed to be a member of the programme's medical team. Though the researcher took pains to rectify this misunderstanding, it may have influenced aspects of the sampling and quality of responses. Patients unwilling to disclose psychological information to $U$ Turn Medical staff may not have known that the study would be conducted by an external researcher, who was bound by researcher-participant confidentiality. Volunteers may have been similarly misinformed and thus they restricted their responses to more socially desirable or edited versions.

Lastly, contextual constraints and limits on the research process require due consideration. Data collection at the Sport and Exercise Medicine (SEM) clinic was limited by patient intake into the U Turn Medical programme, which was infrequent at times. In addition, staff may have inadvertently used the recruitment stage as more of a referral system, often only remembering to introduce the study to patients who had mentioned psychological problems early in the intake process. This may have created a bias in the cohort who contributed to this investigation.

\section{Conclusion}

Noncommunicable disease events and diagnoses are undoubtedly traumatic for many, and may require much resilience to overcome them. The study promotes the use of individualised assessment strategies to ascertain the nature and perceived importance of any trauma or related barriers experienced by patients with NCDs. Specifically, using semistructured interviews, the study allowed for in-depth descriptions of NCDs patients' perceptions of and responses to their illness, as well as their recovery process. By providing a flexible and individualised investigative approach the study was able to yield clinically relevant psychosocial insights of patients with this complex condition. It is hoped that such techniques be included in the routine assessment of patients with NCDs which, in turn, will further the development of patient-centred interventions in these and other disease cohorts.

Conflict of interest: One of the authors (WD) co-directed the $U$ Turn Medical programme at the time of data collection and analysis.

\section{References}

1. World Health Organisation. Global Status Report on Noncommunicable Diseases 2010. (Alwan A, ed.). Geneva: World Health Organisation; 2011. http://www.cabdirect.org/abstracts/20113168808.html. Accessed November 2, 2014

2. Matheson GO, Klügl M, Engebretsen L, et al. Prevention and management of non-communicable disease: the IOC consensus statement, Lausanne 2013. Br J Sports Med 2013;47(16):1003-1011. doi:10.1136/bjsports-2013-093034.

3. Welstand J, Carson A, Rutherford P. Living with heart failure: an integrative review. Int J Nurs Stud 2009;46(10):1374-1385. doi:10.1016/j.ijnurstu.2009.03.009.

4. Yu DSF, Lee DTF, Kwong ANT, et al.. Living with chronic heart failure: a review of qualitative studies of older people. J Adv Nurs 2008;61(5):474-483. doi:10.1111/j.1365-2648.2007.04553.x.

5. Jeon $\mathrm{Y}-\mathrm{H}, \mathrm{Kraus} \mathrm{SG}$, Jowsey T, et al. The experience of living with chronic heart failure: a narrative review of qualitative studies. BMC Health Serv Res 2010;10(77):1-9. doi:10.1186/1472-6963-10-77.

6. Corcoran $\mathrm{KJ}$, Jowsey $\mathrm{T}$, Leeder SR. One size does not fit all: the different experiences of those with chronic heart failure, type 2 diabetes and chronic obstructive pulmonary disease. Aust Heal Rev 2013;37(1):19-25. doi:10.1071/AH11092.

7. Gregory S, Bostock Y, Backett-Milburn K. Recovering from a heart attack: a qualitative study into lay experiences and the struggle to make lifestyle changes. Fam Pract 2006;23(2):220-225. doi:10.1093/fampra/cmi089.

8. Neubeck L, Freedman SB, Clark AM, et al.. Participating in cardiac rehabilitation: a systematic review and meta-synthesis of qualitative data. Eur J Prev Cardiol 2012;19:494-503. doi:10.1177/1741826711409326.

9. Derman W, Schwellnus M, Hope F, et al. Description and implementation of U-Turn Medical, a comprehensive lifestyle intervention programme for chronic disease in the sport and exercise medicine setting: pre-post observations in 210 consecutive patients. $\mathrm{Br} \quad \mathrm{J}$ Sports Med 2014;48(17):1316-1321. doi:10.1136/bjsports-2014-093814.

10. Britten N. Qualitative interviews in medical research. BMJ 1995;311(6999):251-253.

11. Potter J, Hepburn A. Qualitative interviews in psychology: problems and possibilities. Qual Res Psychol 2005;2(4):281-307. doi:10.1191/1478088705qp045oa.

12. Braun V, Clarke V. Using thematic analysis in psychology. Qual Res Psychol 2006;3(2):77-101. doi:10.1191/1478088706qp063oa.

13. Boyatzis RE. Transforming qualitative information: Thematic analysis and code development. London: Sage; 1998.

14. de Sousa Pinto JM, Martín-Nogueras AM, Morano MTAP,et al.. Chronic obstructive pulmonary disease patients' experience with pulmonary rehabilitation: a systematic review of qualitative research. Chron Respir Dis 2013;10(3):141-157. doi:10.1177/1479972313493796.

15. Coulter A, Ellins J. Effectiveness of strategies for informing, educating, and involving patients. BMJ 2007;335(7609):24-27. doi:10.1136/bmj.39246.581169.80. 